\title{
Detection and spatial mapping of conductive filaments in metal/oxide/metal cross-point devices using a thin photoresist layer
}

Cite as: Appl. Phys. Lett. 114, 062901 (2019); https://doi.org/10.1063/1.5084060

Submitted: 03 December 2018 . Accepted: 24 January 2019 . Published Online: 12 February 2019

Shimul Kanti Nath, Sanjoy Kumar Nandi (D), Shuai Li, and Robert Glen Elliman (i)
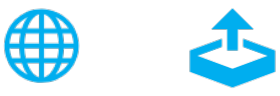

\section{ARTICLES YOU MAY BE INTERESTED IN}

Improvement of memristive properties in CuO films with a seed Cu layer

Applied Physics Letters 114, 061602 (2019); https://doi.org/10.1063/1.5079876

Effect of thickness of metal electrode on the performance of $\mathrm{SiN}_{\mathbf{X}}$-based resistive switching devices

Applied Physics Letters 114, 042102 (2019); https://doi.org/10.1063/1.5062597

Dielectric function and band structure of $\mathrm{Sn}_{1-x} \mathrm{Ge}_{\mathrm{x}}(\mathrm{x}<0.06)$ alloys on InSb

Applied Physics Letters 114, 062102 (2019); https://doi.org/10.1063/1.5086742

Applied Physics Reviews Now accepting original research 


\title{
Detection and spatial mapping of conductive filaments in metal/oxide/metal cross-point devices using a thin photoresist layer
}

\author{
Cite as: Appl. Phys. Lett. 114, 062901 (2019); doi: 10.1063/1.5084060 \\ Submitted: 3 December 2018 - Accepted: 24 January 2019 • \\ Published Online: 12 February 2019
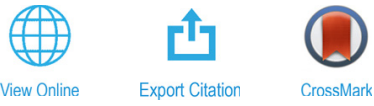

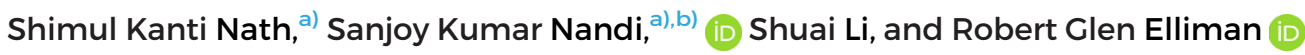

\begin{abstract}
AFFILIATIONS
Department of Electronic Materials Engineering, Research School of Physics and Engineering, The Australian National University, Canberra ACT 2601, Australia
\end{abstract}

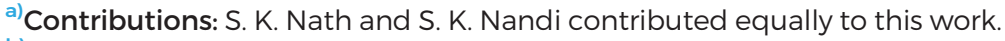

${ }^{\text {b) }}$ Author to whom correspondence should be addressed: sanjoy.nandi@anu.edu.au

\begin{abstract}
A simple means of detecting and spatially mapping volatile and nonvolatile conductive filaments in metal/oxide/metal crosspoint devices is introduced, and its application demonstrated. The technique is based on thermal discolouration of a thin photoresist layer deposited on the top electrode (TE) of the cross-point device and relies on the increase in temperature produced by local Joule heating of an underlying conductive filament. Finite element modelling of the temperature distribution and its dependencies shows that the maximum temperature at the TE/photoresist interface is particularly sensitive to the TE thickness. The technique is demonstrated on $\mathrm{NbO}_{\mathrm{x}}$-based metal-oxide-metal cross-point devices with a $25 \mathrm{~nm}$ thick top (Pt) electrode, where it is used to undertake a statistical analysis of the filament location as a function of device area. This shows that filament formation is heterogeneous; the majority of filaments form preferentially along the TE edge, and the fraction of these increases with the decreasing device area. Transmission electron microscopy of the top and bottom electrode edges is used to explain this observation and suggests that it is due to a reduction in the effective oxide thickness in this region.
\end{abstract}

Published under license by AIP Publishing. https://doi.org/10.1063/1.5084060

Characteristic resistance changes are observed in twoterminal metal-oxide-metal (MOM) structures subjected to large electric fields or current densities and are of interest as the basis of non-volatile memory and neuromorphic computing devices. $^{1-4}$ The resistance changes are commonly mediated by filamentary conduction, either in the form of a semi-permanent filament created by compositional changes in the oxide or as a transient filament created by inhomogeneous current or field distributions (e.g., current bifurcation). Semi-permanent filaments are typically composed of oxygen vacancies created by the generation, drift, and diffusion of oxygen ions during an electroforming step, ${ }^{5,6}$ while transient filaments can self-assemble in materials that exhibit S-type negative differential resistance (NDR) due to current bifurcation, a process in which a uniform current divides into domains of high and low current-densities. ${ }^{7}$ In both cases, the filaments create low resistance paths through the oxide film and can result in a significant temperature rise due to local Joule heating when carrying current.,
Knowledge about the structure, composition, and spatial distribution of filaments is essential for a full understanding of filamentary resistive-switching and for effective modelling and optimisation of associated devices. As a consequence, there is great interest in methods for detecting and characterising conductive filaments. The existence of filamentary conduction is often inferred from area-independent switching characteristics $^{10}$ but has also been verified directly by conductive atomic force microscopy (C-AFM) $)^{11,12}$ and infrared (thermal) microscopy (IR-M). ${ }^{9}$ Compositional analysis of filaments has been undertaken by transmission electron microscopy (TEM) ${ }^{13}$ but this requires pre-knowledge of the filament location and is not suitable for large-scale statistical analysis. Each of these techniques has its advantages and disadvantages, but only IR-M is well suited to large-scale statistical analysis of filament distributions and to the detection of transient filaments.

In this study, we introduce a simple alternative technique for locating volatile or nonvolatile filaments in MOM cross-point 
devices based on thermal discolouration of a thin photoresist layer deposited on the top electrode (TE) of the cross-point device. The technique relies on the increase in temperature at the top-electrode/photoresist interface produced by local Joule heating of an underlying conductive filament and can be used to identify the number and position of filaments. The efficacy of this approach is demonstrated by applying it to filament formation in MOM cross-point devices composed of a $\mathrm{Pt} / \mathrm{Nb}(\mathrm{Cr}) /$ $\mathrm{NbO}_{\mathrm{x}} / \mathrm{Pt}$ heterostructure.

$\mathrm{Pt} / \mathrm{Nb}(\mathrm{Cr}) / \mathrm{NbO}_{\mathrm{x}} / \mathrm{Pt}$ cross-point test structures, as shown schematically in Fig. 1(a), were fabricated using standard photolithographic processing (supplementary material). Both bottom and top electrodes were defined by a photolithographic lift-off process using negative photoresist (MaN 1420). The bottom electrode (BE) consisted of a $5 \mathrm{~nm}$ thick Ti wetting-layer and a $25 \mathrm{~nm}$-thick Pt layer deposited by e-beam evaporation on a thermally oxidized $\mathrm{Si}(100)$ wafer with a $200 \mathrm{~nm}$ thick oxide layer. A $45 \mathrm{~nm} \mathrm{NbO}_{\mathrm{x}}$ dielectric layer was then deposited over the entire wafer, including the lithographically defined bottom electrode, using RF sputtering from an $\mathrm{Nb}_{2} \mathrm{O}_{5}$ target. Top electrodes, consisting of a $10 \mathrm{~nm}$-thick $\mathrm{Nb}$ (or $\mathrm{Cr}$ ) layer and a $25 \mathrm{~nm}$ Pt protective layer, were then deposited by e-beam evaporation. The oxide layer covering the bottom contact pads was then removed by etching to provide a direct electrical contact to the pad. The test-devices had electrode dimensions ranging from $2 \mu \mathrm{m}$ $\times 2 \mu \mathrm{m}$ to $20 \mu \mathrm{m} \times 20 \mu \mathrm{m}$.

The as-deposited $\mathrm{NbO}_{\mathrm{x}}$ films were analysed by grazing incident-angle X-ray diffraction (GIAXRD) and Rutherford backscattering spectroscopy (RBS) to determine their structure and composition. ${ }^{14}$ To simplify the RBS analysis, $\mathrm{NbO}_{\mathrm{x}}$ films were (a)

(c)

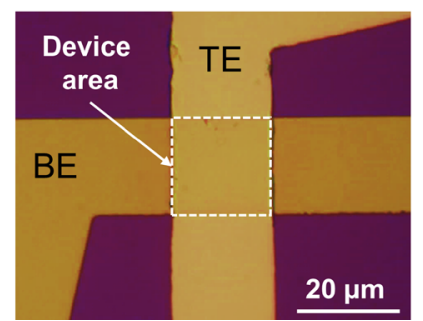

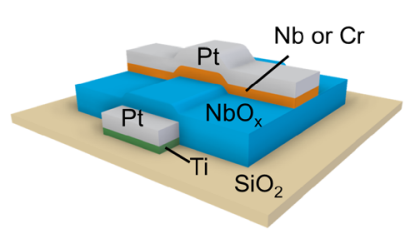

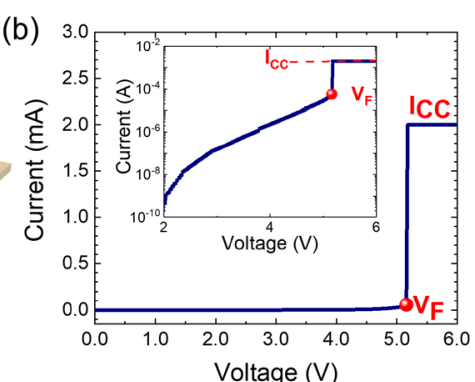

(d)

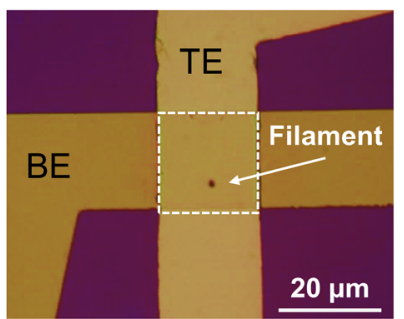

FIG. 1. (a) A schematic of the device cross-point structure and (b) a typical currentvoltage (I-V) characteristic of the electroforming process for a $20 \mu \mathrm{m} \times 20 \mu \mathrm{m} \mathrm{Pt} /$ $\mathrm{Cr} / \mathrm{NbO}_{x} / \mathrm{Pt}$ device. The inset shows a semi-log plot of the I-V characteristic. (c) and (d) Optical microscopy images of the same device before (c) and after (d) the electroforming process. The dark spot on the device after forming indicates the location of a conducting filament. simultaneously deposited on vitreous carbon substrates. These analyses showed that the as-deposited films were amorphous, with a stoichiometry of $\mathrm{NbO}_{\mathrm{x}}$, where $\mathrm{x}=2.60 \pm 0.05$ (i.e., oxygen rich $\mathrm{Nb}_{2} \mathrm{O}_{5}$ ). Electrical measurements were performed using an Agilent B1500A semiconductor parameter analyser attached to a Signatone probe station (S-1160). All the measurements were executed at room temperature under atmospheric conditions by applying voltage on the top electrode, while the bottom electrode was grounded.

The as-fabricated devices were in a highly resistive state $\left(>10^{10} \Omega\right)$ and required a one-step electroforming process to initiate resistive switching characteristics. This was achieved by applying a positive voltage sweep from $0 \mathrm{~V}$ to $+6 \mathrm{~V}$ to the top electrode while limiting the maximum current through the device [compliance current $\left(\mathrm{I}_{\mathrm{CC}}\right)$ ] to $\sim 2 \mathrm{~mA}$ to avoid permanent damage. Figure 1(b) shows typical electroforming characteristics of a $20 \mu \mathrm{m} \times 20 \mu \mathrm{m} \mathrm{Pt} / \mathrm{Cr} / \mathrm{NbO}_{\mathrm{x}} / \mathrm{Pt}$ device. The electroforming voltage, $V_{F}$, is defined as the point at which the current reaches the compliance limit and corresponds to an electric field of $\sim 1$ $\mathrm{MV} / \mathrm{cm}$ for this device, which is consistent with previous studies. ${ }^{10}$ Following the electroforming step, the device resistance was $\sim 5 \mathrm{k} \Omega$, consistent with the formation of a permanent conductive filament.

The high current density associated with filamentary conduction is known to cause a rapid increase in temperature due to local Joule heating. Indeed, in-situ thermal mapping has shown that the surface temperature of the filament can increase by several hundred degrees. ${ }^{9}$ Here, we exploit this temperature increase to thermally denature a thin photoresist layer and record the location of the filament. To achieve this, the crosspoint devices were coated with a thin positive photoresist (AZ 1512HS) layer and a photolithographic step used to reopen access to the device contact pads. The photoresist was then baked for $2 \mathrm{~min}$ in air using a hot plate kept at a temperature of $358 \mathrm{~K}\left(85^{\circ} \mathrm{C}\right)$. Figure 1 (c) shows an image of a $20 \mu \mathrm{m} \times 20 \mu \mathrm{m}$ cross-point before electroforming, and Fig. 1(d) shows an image of the same device after electroforming. It is immediately evident that electroforming creates a dark spot in the photoresist, clearly identifying the filament location. Analysis of more than 176 devices showed that electroforming produced a single filament in most cases, with only a few showing multiple filaments. This is in agreement with previous results, indicating the dominance of single filament switching. $8,15,16$

To determine the relationship between the temperature of the filament and that at the top-electrode/photoresist interface, a finite element model of the device structure and filamentary Joule heating was constructed using the COMSOL package. The details of the model are presented in the supplementary material. Suffice it to say that the simulation used a 2Daxiosymetric model of the device structure and the filamentary conduction process to calculate the steady-state temperature distribution in the device as a function of applied current.

Figure 2(a) shows the calculated temperature distribution in the active volume of the device for a current of $2.0 \mathrm{~mA}$ and clearly shows the temperature increase at the top electrode due to Joule heating of the filament in the underlying $\mathrm{NbO}_{\mathrm{x}}$ layer. This is shown more quantitatively in Fig. 2(b) which plots the 
(a) Axis of rotation

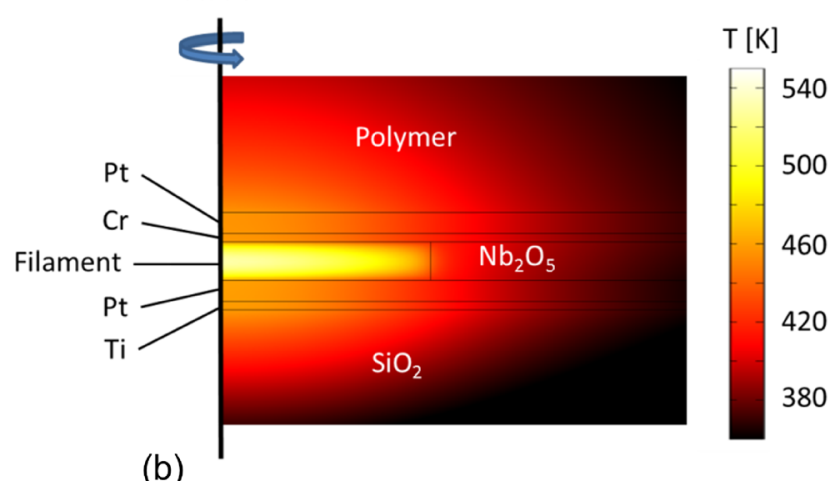

(b)

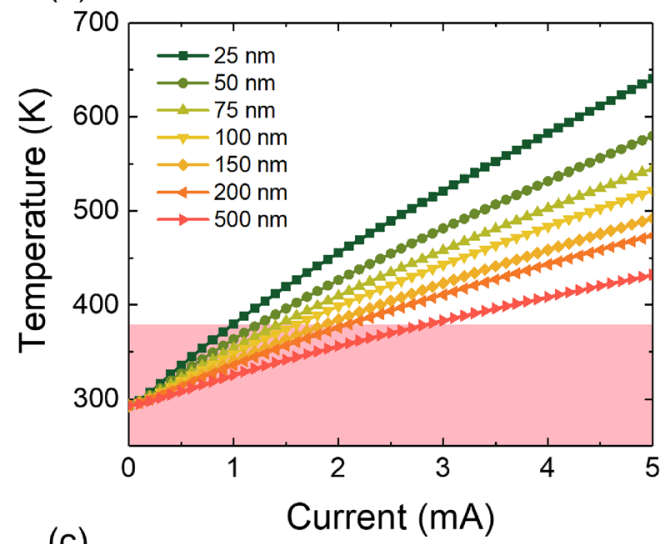

(c)

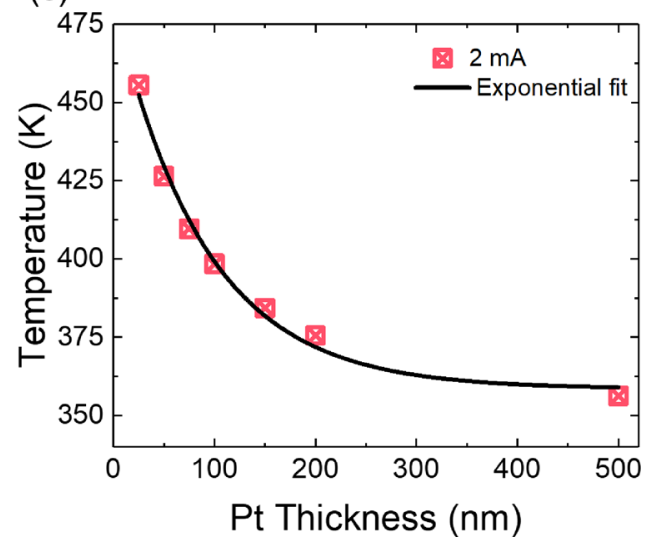

FIG. 2. (a) Temperature distribution in the active volume of the device for a current of 2.0 mA. The depicted structure presents a cross-section of an axisymmetric model with the axis of rotation shown. For reference, the thickness of the oxide layer is $45 \mathrm{~nm}$ and the width of the conductive filament is $250 \mathrm{~nm}$. (b) Maximum temperature at the top-electrode/photoresist interface as a function of device current and top electrode (Pt) thickness. (c) Maximum temperature at the top-electrode/ photoresist interface as a function of top electrode (Pt) thickness.

maximum temperature at the top-electrode/photoresist interface as a function of device current and top (Pt) electrode thickness. This shows that the temperature is a strong function of both parameters and reaches a value of $456 \mathrm{~K}$ for a current of $2.0 \mathrm{~mA}$ and an electrode thickness of $25 \mathrm{~nm}$, more than sufficient to modify the photoresist. However, as shown in Fig. 2(c), the maximum temperature decreases rapidly with increasing top electrode thickness, decreasing from $456 \mathrm{~K}$ to $398 \mathrm{~K}$ as the electrode thickness increases from $25 \mathrm{~nm}$ to 100 $\mathrm{nm}$. These simulations confirm that the top-electrode/photoresist interface can reach temperatures sufficient to degrade the photoresist but highlight the importance of using thin top electrodes in maximising the sensitivity of the technique, i.e., filament detection would not be possible in the present case if the electrode thickness was increased significantly.

The utility of the proposed filament detection method is demonstrated by using it to study the influence of the device structure on the stochastic nature of electroforming. To this end, the spatial distribution of 48 filaments was recorded for $20 \mu \mathrm{m} \times 20 \mu \mathrm{m}\left(400 \mu \mathrm{m}^{2}\right)$ devices using images such as those shown in Fig. 1(d). The results are plotted in Fig. 3(a) and show that a significant fraction of filaments form at the electrode edges. Moreover, there seems to be a preference for filament formation along the top-electrode edge rather than the bottom electrode edge. To further investigate this point, a similar analysis was performed on devices of different area and is summarised in Fig. 3(b). This clearly shows that the fraction of filaments formed at the device edges is a strong function of device area and that there is a stronger preference for filament nucleation at the top-electrode edge in smaller devices; for the $20 \mathrm{um} \times 20$ um $\left(400 \mu \mathrm{m}^{2}\right)$ devices, $40 \%$ of filaments formed at electrode edges, with $\sim 30 \%$ forming at the top electrode (TE) edges and $\sim 10 \%$ at the bottom electrode (BE) edges, while for $5 \mu \mathrm{m} \times 5 \mu \mathrm{m}$ devices, the edge fraction increases to $75 \%$, with $50 \%$ at the top edge and $25 \%$ at the bottom edge. These results clearly show that device-related inhomogeneities can play an important role in filament formation. Such effects need to be understood for a full interpretation of area-dependent scaling effects.

For example, the forming voltage for randomly distributed filaments is expected to decrease with the increasing device area and to have a dependence of the form ${ }^{17,18}$

$$
\mathrm{V}_{f}=\mathrm{C}_{1}-\mathrm{C}_{2} \ln \left(\frac{\mathrm{A}}{a^{3}}\right)
$$

where $V_{f}$ is the forming voltage, $C_{1,2}$ are the constants associated with statistics of the forming process, $a^{3}$ is a characteristic material volume, and A is the device area. Figure 3(c) shows the area dependence for the devices in this study, clearly demonstrating that the forming voltage and dispersion of the forming voltage increase with decreasing area, most likely due to heterogeneous nucleation of filaments at the electrode edges.

Given that defect formation depends exponentially on the local electric field, the probability for forming is expected to increase at the electrode edges and to be strongly influenced by variations in the oxide thickness, such as those that might arise from deposition onto the non-planar bottom electrode structure. ${ }^{19}$ In this context, preferential forming at the top electrode edge seems counter-intuitive. To better understand how this might arise, cross-sectional TEM images were taken at the top and bottom edges as shown in Fig. 4. These show that the metal electrode layers extend beyond the nominal photolithographic mask edges and into the region where the photoresist is 
(a)

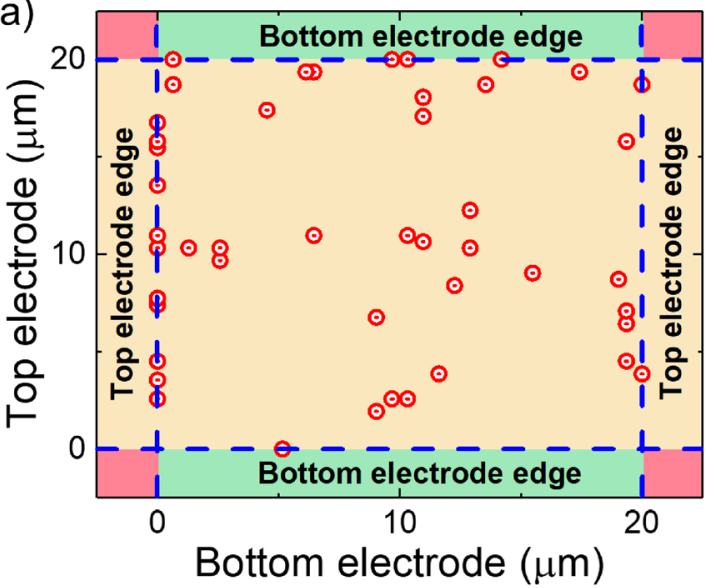

(b)
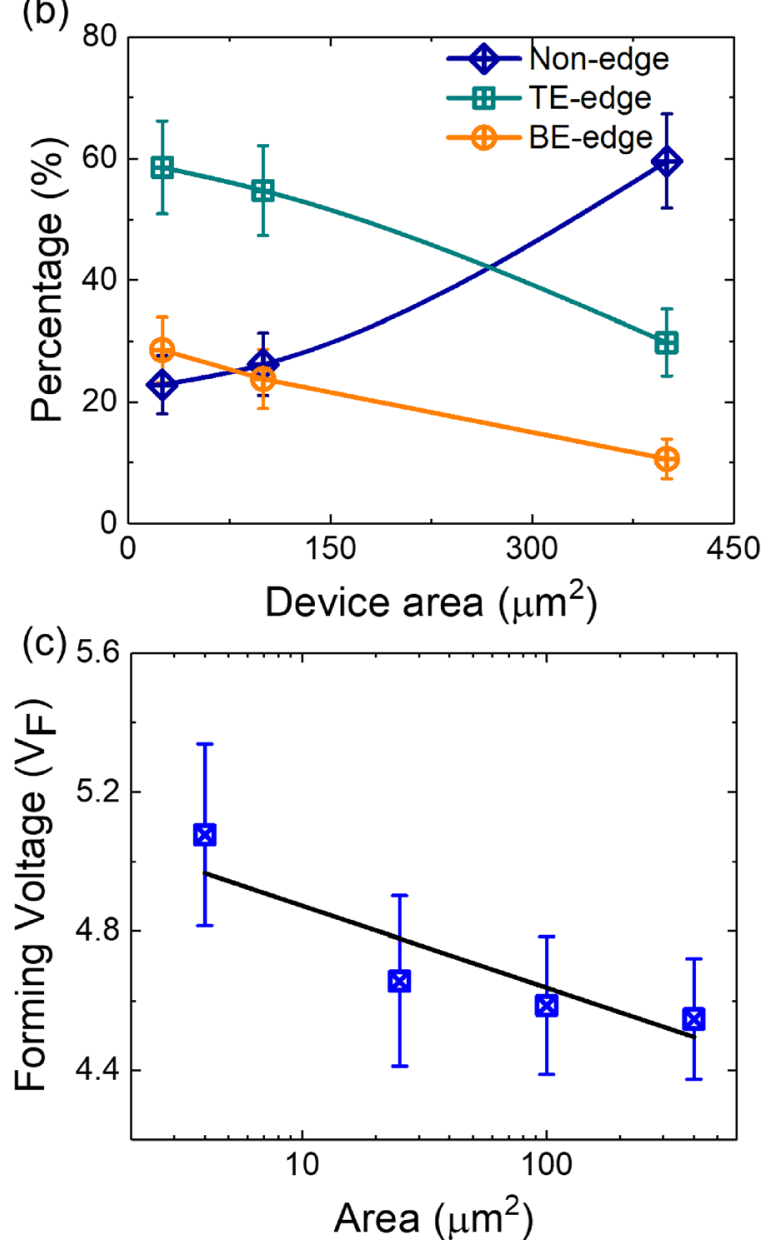

FIG. 3. (a) Schematic of the $20 \mu \mathrm{m} \times 20 \mu \mathrm{m}$ cross-point device showing the measured location of filaments (red dots) for 48 different devices. The overlaid dashed lines here show the position of the electrode edges in the cross-point structure. (b) Percentage of devices formed at the edge of the cross-point as a function of device area. The statistical analysis represents 48 different devices of $20 \mu \mathrm{m} \times 20 \mu \mathrm{m}$ size, 56 devices of $10 \mu \mathrm{m} \times 10 \mu \mathrm{m}$ size, and 72 devices of $5 \mu \mathrm{m} \times 5 \mu \mathrm{m}$ size. (c) Variation of breakdown voltage as a function of device area. (a)

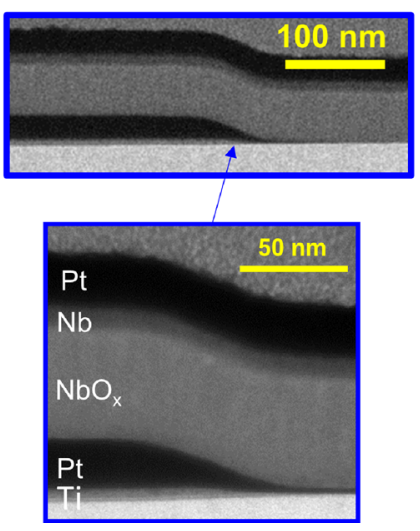

(b)

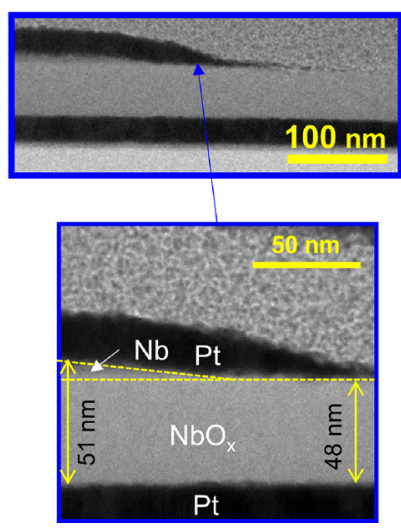

FIG. 4. Cross-sectional TEM of the cross-point device at (a) bottom edge and (b) top edge.

undercut to facilitate the lift-off process, an effect that is clearly evident in Fig. 4(a). The extent of metal penetration increases with increasing metal thickness, and is therefore greater for the Pt layer than for the $\mathrm{Ti}$ and $\mathrm{Nb}(\mathrm{Cr})$ wetting layers. This produces a region at the edge of the electrodes where Pt is in direct contact with the $\mathrm{NbO}_{\mathrm{x}}$ layer, as shown in Fig. 4(b). This is significant because the top $\mathrm{Nb}(\mathrm{Cr})$ wetting layer is expected to react with $\mathrm{NbO}_{\mathrm{x}}$ to extend the effective oxide thickness. The oxide layer is therefore thinner at the top-electrode edge where it is in direct contact with $\mathrm{Pt}$, possibly explaining why the majority of filaments form in this region.

In summary, we have demonstrated a simple technique to detect and spatially map volatile and nonvolatile conductive filaments in micron-scale metal/oxide/metal cross-point devices based on thermal discolouration of a thin photoresist layer. Finite element modelling showed that the temperature of the top-electrode/photoresist interface can easily exceed that required to discolour photoresist but that the maximum temperature is a strong function of electrode thickness. The application of this technique to metal-oxide-metal cross-point devices confirmed that electroforming generally created one dominant filament and showed that filament formation was heterogeneous, with filaments preferentially forming along the edge of the top electrode. TEM analysis of the top and bottom electrode edges suggested that this was due to a reduction of the effective oxide thickness in this region. While further work is required to confirm this hypothesis, this study serves to illustrate the efficacy of the proposed filament mapping technique for understanding such effects. We also note that the sensitivity and spatial resolution of the technique could be improved by using a more temperature-sensitive polymer and further reducing the top electrode thickness.

See supplementary material for the details of device fabrication, the schematic of the filament location of $10 \mu \mathrm{m} \times 10 \mu \mathrm{m}$ and $5 \mu \mathrm{m} \times 5 \mu \mathrm{m}$ cross-point devices, and the supportive data for finite element modelling. Additional TEM images also included to show the undercut region of a $\mathrm{Pt} / \mathrm{HfO}_{2} / \mathrm{Pt}$ device. 
The authors acknowledge financial support from the Australian Research Council (ARC) Discovery. We would also like to acknowledge the ACT node of the Australian National Fabrication Facility (ANFF) for access to their research equipment and expertise. The authors also acknowledge the facilities, and the scientific and technical assistance, of the Australian Microscopy and Microanalysis Research Facility at the Centre of Advanced Microscopy, The Australian National University.

\section{REFERENCES}

${ }^{1} \mathrm{C}$. Dias, J. Ventura, and P. Aguiar, Advances in Memristors, Memristive Devices and Systems (Springer, 2017), pp. 305-342.

${ }^{2}$ A. Beaumont, J. Leroy, J.-C. Orlianges, and A. Crunteanu, J. Appl. Phys. 115(15), 154502 (2014).

${ }^{3}$ S. Li, X. Liu, S. K. Nandi, D. K. Venkatachalam, and R. G. Elliman, Appl. Phys. Lett. 106(21), 212902 (2015).

${ }^{4}$ S. Li, X. Liu, S. K. Nandi, D. K. Venkatachalam, and R. G. Elliman, Nanotechnology 28(12), 125201 (2017).

${ }^{5}$ S. K. Nandi, X. Liu, S. Li, D. K. Venkatachalam, K. Belay, and R. G. Elliman, in Proceedings of 2014 Conference on Optoelectronic and Microelectronic Materials Devices (COMMAD), Perth, Australia, 14-17 December 2014 (IEEE, 2014), pp. 290-293.
${ }^{6}$ S. K. Nandi, X. Liu, D. K. Venkatachalam, and R. G. Elliman, Phys. Rev. Appl. 4(6), 064010 (2015).

${ }^{7}$ B. Ridley, Proc. Phys. Soc. 82(6), 954 (1963).

${ }^{8}$ S. Kumar, Z. Wang, N. Davila, N. Kumari, K. J. Norris, X. Huang, J. P. Strachan, D. Vine, A. D. Kilcoyne, and Y. Nishi, Nat. Commun. 8(1), 658 (2017).

${ }^{9}$ M. Uenuma, Y. Ishikawa, and Y. Uraoka, Appl. Phys. Lett. 107(7), 073503 (2015).

${ }^{10}$ L. Shuai, L. Xinjun, N. Sanjoy Kumar, and E. Robert Glen, Nanotechnology 29(37), 375705 (2018)

${ }^{11}$ M. Lanza, U. Celano, and F. Miao, J. Electroceram. 39(1-4), 94-108 (2017).

${ }^{12}$ M. Lanza, Materials 7(3), 2155-2182 (2014).

${ }^{13}$ G. A. Gibson, S. Musunuru, J. Zhang, K. Vandenberghe, J. Lee, C.-C. Hsieh, W. Jackson, Y. Jeon, D. Henze, and Z. Li, Appl. Phys. Lett. 108(2), 023505 (2016).

${ }^{14}$ M. Vos, X. Liu, P. Grande, S. Nandi, D. Venkatachalam, and R. Elliman, Nucl. Instrum. Methods Phys. Res., Sect. B 340, 58-62 (2014).

${ }^{15}$ J. P. Strachan, D. B. Strukov, J. Borghetti, J. J. Yang, G. Medeiros-Ribeiro, and R. S. Williams, Nanotechnology 22(25), 254015 (2011).

${ }^{16}$ J. P. Strachan, J. J. Yang, R. Münstermann, A. Scholl, G. MedeirosRibeiro, D. R. Stewart, and R. S. Williams, Nanotechnology 20(48), 485701 (2009).

17J. Wang and S. S. Nonnenmann, J. Mater. Sci. 52(11), 6469-6475 (2017).

${ }^{18}$ A. Chen, IEEE Electron Device Lett. 35(1), 57-59 (2014).

${ }^{19}$ J. Leroy, A. Crunteanu, A. Bessaudou, F. Cosset, C. Champeaux, and J.-C. Orlianges, Appl. Phys. Lett. 100(21), 213507 (2012). 